\title{
Collaboratory Testbed Partnerships as a Knowledge Capture Challenge
}

\author{
Mary A. Keeler \\ Center for Advanced Research Technology \\ in the Arts and Humanities \\ University of Washington \\ Seattle, Washington 98117, USA \\ 206-784-4267 \\ mkeeler@u.washington.edu
}

\author{
Heather D. Pfeiffer \\ Department of Computer Science \\ New Mexico State University \\ Las Cruces, New Mexico \\ 88003-8001, USA \\ 505-496-3502 \\ hdp@cs.nmsu.edu
}

\begin{abstract}
The difficulty of maintaining effective testbed partnerships, among users of computer-based tools and builders of those tools, has been an obstacle to the success of science collaboratories. Testbed partnerships are as essential for knowledge-tool advancement as that advancement is to the support of effective partnerships, because knowledge tools must be instituted in a context that truly augments (rather than merely tries to replicate) human inference in collaborative contexts. We propose a game framework for engaging content-tool users and context-tool developers in testbed partnerships, and an architecture for modular knowledge-tool integration, as initial steps in the effective advancement of knowledge capture and improvement of testbed partnerships.
\end{abstract}

\section{Categories and Subject Descriptors H.3.0 [INFORMATION STORAGE AND RETRIEVAL]: General}

\section{General Terms}

Management, Design, Experimentation, Human Factors.

\section{Keywords}

Collabortories, Testbeds, Games, Hypotheses, Integration, and Modular

\section{INTRODUCTION}

Many promising knowledge tools are stranded at the end of the research and development cycle, waiting to be found by some user community. Meanwhile, user communities are struggling to find tools powerful enough to augment their increasing collaborative, intellectual work. We conceive the gap between developers and potential users of tools as a knowledge-capture problem: users' knowledge of their work domain must correlate with technologists' knowledge of what their tools can do. The collaboratory-testbed method of technology customization and transfer is an appropriate solution, if testbeds can be developed as they were originally conceived [see 1:14].

Copyright is held by the author/owner(s).

K-CAP'05, October 2-5, 2005, Banff, Alberta, Canada.

ACM 1-59593-163-5/05/0010.
Testbeds were to support partnerships between users and technologists in exploring the utility of various technical approaches by which a scientist user-community could take advantage of emerging technology. In virtual testbed contexts, users could remotely collaborate to examine, calibrate, validate, and interpret some particular data content, while their technologist partners observe their work and introduce coordination technologies in experimental versions integrated for use in actual working contexts. The appropriate components of systems architecture and an array of tools for data access and communication would be designed and developed within a program of prototyping, testing, and evaluation that could support continuous improvement. Testbed participants were to be a sample group from the user community for whom data content manipulation and collaboration context support technology would be developed, and they were to be committed to work in partnerships with committed technologists.

Partners in the testbed mode of development must consider: the nature of the content data, the requirements of computing context needed to manipulate the content, and how the context might be improved in order to improve the content development, collaboratively. Testbeds must coordinate among all collaborators (users and technologists) who are geographically distributed, who necessarily represent a range of disciplines, and who are committed to a continuing study of what might improve the quality of both data content and testbed context. Their ability to institute this self-critically controlled conduct - as the directing agency of user-tool co-evolution-will determine whether they can engage in effective partnerships. Based on game theoretical findings of how such cooperation and coordination capabilities evolve, we propose a game framework for instituting the self-critical conduct required in testbed partnerships.

\section{GAMES FOR ENGAGING TESTBED PARTNERSHIPS}

Testbed partnerships and tool evolution can be conceptualized in a game theory framework [see 2], with tool users engaged in game-playing, while tool developers engage in tournaments to capture the variety of possible tool strategies. The users' game would involve their 
particular content knowledge and the collaborative evolution of its concepts. A possible framework for such a game is Robert Brandom's model of discursive practice, which treats concepts as norms. Concepts are nodes in an evolving inferential network of related concepts, which determines "what counts as a reason for particular beliefs, claims, and intentions," in the same way that rules determine the correctness of moves in a game [3:25; $4: 243]$. Brandom's model identifies conceptual content in an expression by whether it can play a role in the inferential game of "making claims and giving and asking for reasons." We give beliefs, desires, and intentions conceptual content when we ask such pragmatic questions as "under what circumstances would what is believed, desired, or intended be true?" [4:158]. The representational use of propositional concepts relies on the social structure of their inferential articulations in the game of giving and asking for reasons [see 4:183]. Conceptual content is collaborative.

In the game framework, each player's commitments are asserted as beliefs and the supporting entitlements are reasons for those beliefs. Players express these commitments and entitlements to make explicit their implicit assumptions in the "inferentially articulated network" that the game requires. Players in a testbed game must report their beliefs (or hypotheses) in conditional form, to make explicit what is not otherwise made explicit in their ordinary discourse concerning whatever they consider significant in their ongoing work. By using this propositional form in natural language (which need not be constrained to strict "if-then" statements) each player asserts hypotheses to engage in collaborative discourse. The game framework then enables them to formulate valid hypotheses collaboratively, to explain the implications of the data studied as the content of their work [see 5 for detailed scenario].

Using what Brandom calls the normative fine structure of rationality (NFS), the players can rely on the mechanism of the game to help them focus on and clarify the complexities of inference and conceptual content in their work's collaborative evolution. Incompatibilities that emerge mark possibly missing hypothetical inferences that should direct further inquiry. Meanwhile commitment-preserving inferences trace the implications of validly related claims, and entitlement-preserving inferences record the tested reliability of claims with respect to any data under their collaborative investigation.

The game is a method of "harmonizing" the implicit elements of their collaborative conceptual content, by pinpointing incompatibilities to be resolved. While each attempts to maximize individual scores (the count of each player's legitimate claims), players must minimize the score of incompatibility entailments to improve their collaborative hypotheses, as the ultimate objective of the game. The effect is similar to judges formulating principles of common law by codifying prior practice as represented in precedent, to express explicitly as a rule what was implicit in that precedent, for regulative authority in subsequent practice [see 4:75-76]. That is also the effect of good standards-making in technology development.

\section{CONCLUSIONS}

The testbed method of user-developer partnerships, based on component interoperability, should respond to evolving and diverse user requirements. In testbeds, tool users and technology developers can participate in refining and validating both the functionality of the tools and their own habits of behavior. Knowledge tool developers can carefully consider how their systems actually work in particular contexts of operation, while users have the chance to become comfortable with new systems gradually by participating in their development. Based on findings from game theory and Brandom's model of discursive practice as the game of "asking for and giving reasons," we propose that testbed games could be created to build the partnerships and the knowledge tools needed for effective collaboratory testbeds. In collaboratory testbed games, with knowledge tools as integratable components, users and developers can explicitly modify their habits along with tool improvements, in co-evolution.

\section{REFERENCES}

[1] Lederberg, J. and Uncaphar, K. (Eds.) Towards a National Collaboratory: Report of an invitational workshop at the Rockefeller University, March 13-15, 1989 (including W.A. Wulf's white paper: The National Collaboratory).

[2] Axelrod, R. The Evolution of Cooperation. Basic Books, 1984.

[3] Keeler, M. Using Brandom's Framework to Do Peirce's Normative Science. In, Wolff, K.E., Pfeiffer, H.D. and Delugach, H.S. (Eds.), Conceptual Structures at Work, LNAI 3127, Springer-Verlag, 2004, pp. 37-53.

[4] Brandom, R. Articulating Reasons: An Introduction to Inferentialism. Harvard University Press, Cambridge, MA, 2000.

[5] Keeler, M.A. and Pfeiffer, H.D. Games of Inquiry for Collaborative Concept Structuring. In Proceedings of the $13^{\text {th }}$ International Conference on Conceptual Structures (ICCS'05) (Kassel, Germany, July 17-22, 2005), in press. 